\title{
Understanding platform businesses in the food ecosystem
}

Area of research interest: Emerging challenges and opportunities

Study duration: 2021-01-01

Project status: Completed

Authors: Lucy Hart

Conducted by: Foundry4

Date published: 9 February 2022

DOI: https://doi.org/10.46756/sci.fsa.puh821

\section{Background}

The Food Standards Agency (FSA) is responsible for public health in relation to food in England, Wales and Northern Ireland. It makes sure that people can trust that the food they buy and eat is safe and is what it says it is.

As part of this responsibility, the FSA works to understand the continuing evolution of the food landscape to identify opportunities to improve standards of food safety and/or authenticity. As well as any new or magnified risks from which consumers should be protected.

One area that has evolved rapidly is that of digital platforms in the food and drink industry. Consumers are increasingly purchasing food via third party intermediaries, known as 'aggregators', from a range of vendors. Digital platforms remain a relatively new concept, with many launching in the past decade. As such, there has been a knowledge gap in government about how these platforms work and how they impact the landscape in which they operate.

\section{Research approach}

Foundry4 carried out discovery research exploring digital platforms within the food landscape to help narrow this knowledge gap and enable the FSA to make informed decisions in the coming years.

\section{Results}

The project colours in the food platform picture at a more granular level to enable a shared understanding of what is meant by 'platform'.

The project also drafts hypotheses for where and how the FSA may want to intervene, to promote positive behaviours around food being safe and what it says it is.

Research report

PDF

View Understanding platform businesses in the food ecosystem as PDF(Open in a new window) (441.34 KB) 